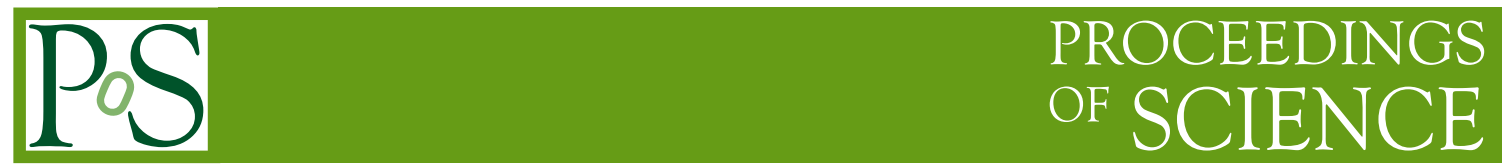

\title{
Initial conditions in heavy ion collisions: from RHIC to the LHC
}

\section{Javier L Albacete*}

IPNO, Université Paris-Sud, CNRS/IN2P3, 91406 Orsay, France,

IPhT, CEA/Saclay, 91191 Gif-sur-Yvette cedex, France.

E-mail: albacetedipno.in2p3.fr

I present a brief discussion of the different approaches to the study initial state effects in heavy ion collisions performed at RHIC and the LHC with emphasis in the Color Glass Condensate approach.

The 2011 Europhysics Conference on High Energy Physics-HEP 2011,

July 21-27, 2011

Grenoble, Rhône-Alpes France

${ }^{*}$ Speaker. 


\section{Introduction}

The studies of initial state effects in heavy ion collisions aim at providing a full dynamical description of the colliding system at early times, before the eventual thermalization of the system and the formation of a Quark Gluon Plasma (QGP). The importance of these studies is manyfold: On the soft sector they determine the initial conditions (energy and entropy density, initial spatial anisotropy etc ) for the subsequent hydrodynamical evolution of the system. On the hard probes sector, they provide a benchmark to quantify final state effects due to the presence of a QGP.

$\mathrm{A}$ main lesson learnt from experimental data collected in $\mathrm{Au}+\mathrm{Au}$ and $\mathrm{Pb}+\mathrm{Pb}$ collisions at RHIC and the LHC respectively is that bulk particle production in ion-ion collisions is very different from a simple superposition of nucleon-nucleon collisions. This is evident in terms of the measured charged particle multiplicities, which exhibit a strong deviation from the scaling with the number of nucleon-nucleon collisions: $\frac{d N^{A A}}{d \eta}(\eta=0) \ll N_{\text {coll }} \frac{d N^{A A}}{d \eta}(\eta=0)$. This observation leads to the conclusion that strong coherence effects among the constituent nucleons must be present during the collisions process. Indeed, any of the phenomenological models who successfully describe data include strong coherence effects, either at the level of the wave function and also at the level of primary particle production. To the first category correspond the nuclear shadowing (in a partonic language) or the percolation and string fusion (in non-perturbative approaches). In both cases, when different constituents, whichever the degrees of freedom chosen are, overlap in phase space according to some geometric criterium, recombination of such constituents happen, thus reducing the total number of scattering centers entering the collision process. Similar phase-space arguments motivate the implementation of energy-dependent cut-offs to regulate independent particle production from different sources, normally a working hypothesis in most Monte Carlo event generators for heavy ion collisions.

\section{The Color Glass Condensate approach.}

The CGC attempts at providing a complete, QCD-based, dynamical description of the coherence effects discussed above (for a review see, e.g., [12]). On one hand, the gluon shadowing is taken into account through non-linear renormalization group equations, the BK-JIMWLK equations, that describe the change in hadron structure towards small Bjorken- $x$ (equivalently, higher collision energies). The non-linear terms in the BK-JIMWLK equations reflect the probability of gluon-gluon recombination processes in the high density regime. Those terms ensure unitarity of the theory and tame the growth of gluon densities towards small- $x$. They also imply the emergence of a dynamical transverse momentum scale, the saturation scale $Q_{s}$, such that gluon modes with transverse momentum $k_{t} \leq Q_{s}(x)$ are in the saturation regime [4]. Due to the complexity of the B-JIMWLK equations, in phenomenological works it is more feasible to solve the BK equation, more tractable numerically, which corresponds to their large- $N_{c}$ limit. It reads

$$
\frac{\partial \mathscr{N}(r, Y)}{\partial Y}=\int d^{2} \mathbf{r}_{\mathbf{1}} K^{\mathrm{run}}\left(\mathbf{r}, \mathbf{r}_{1}, \mathbf{r}_{2}\right)\left[\mathscr{N}\left(r_{1}, Y\right)+\mathscr{N}\left(r_{2}, Y\right)-\mathscr{N}(r, Y)-\mathscr{N}\left(r_{1}, Y\right) \mathscr{N}\left(r_{2}, Y\right)\right]
$$

where $\mathscr{N}(r, Y)$ is the dipole scattering amplitude on a dense target, $Y=\ln x_{0} / x$ the rapidity, $r$ the dipole transverse size and $r_{2}=r-r_{1}$. It turns out that running coupling effects can be incorporated 
to the evolution equation through just a modification of the evolution kernel, referred to as $K^{\text {run }}$ in Eq. (2.1) [4]. Finally, Eq. (2.1) needs to be suplemented with initial conditions, which can be choosen to be of the McLerran-Venugopalan type [17]. This introduces two free parameters: The value $x_{0}$ where the evolution starts and the initial saturation scale $Q_{0}$. Finally, the nuclear unintegrated gluon distribution entering the different production processes discussed below is related to the dipole amplitude in Eq. (2.1) through a Fourier transform:

$$
\tilde{N}(x, k)=\int d^{2} \mathbf{r} e^{-i \mathbf{k} \cdot \mathbf{r}}\left[1-\mathscr{N}\left(r, Y=\ln \left(x_{0} / x\right)\right)\right],
$$

\section{Multiplicities}

Two main features of the new LHC data $[2,1]$ can be highlighted: First, the energy dependence of mid-rapidity multiplicities in $\mathrm{A}+\mathrm{A}$ collisions is well reproduced by a power law, $d N^{c h} / d \eta(\eta=0) \sim s^{0.15}$. This observation seems to rule out the logarithmic trend observed for lower energies data and is in generic agreement with pQCD based approaches. Second, the centrality dependence of multiplicities is, up to an overall scale factor, very similar to the one observed at RHIC. This suggests a factorization of the energy and centrality dependence of the multiplicities, which, in turn, admits a natural explanation in the CGC formalism. There, mid-rapidity multiplicities rise proportional to the saturation scale of the colliding $\mathrm{n}$ uclei which, in a first approximation, is proportional to the local nuclear density or, equivalently, to the number of participants:

$$
\frac{d N}{d \eta}(\eta=0) \sim Q_{s}^{2}(x, b) \sim \sqrt{s}^{\lambda} N_{\text {part }} .
$$

Fig. 1 (right) includes comparison of different Monte Carlo $[9,8]$ and saturation based calculations done in the framework of $k_{t}$-factorization ([13]): $[14,7,3]$ to LHC data on $\mathrm{Pb}+\mathrm{Pb}$ collisions. A best description of data is given by the MC-rcBK approach of [3] and in the MC HIJING approach [9], which includes a very strong impact parameter dependent shadowing. Aside of implementation details different CGC approaches differ mainly in their input for the ugd's: analytical models [14], models constrained from $\mathrm{e}+\mathrm{p}$ and $\mathrm{e}+\mathrm{A}$ data [7] or solutions from the running coupling BK equation. These choices lead to slightly varying effective values of the exponent $\lambda \sim 0.2 \div 0.3$ in (3.1) and partially explain the spread of saturation based predictions. Other distinguishing feature among saturation models is the treatment of impact parameter dependence. It varies from describing the nucleus by a single, average saturation scale, to mean field approaches with explicit b-dependence, to Monte Carlo methods. The latter are the best suited to account for the fluctuations in the initial geometry of the collision, a crucial ingredient for the subsequent analysis of multiparticle correlations, flow, etc. Presently there are two variants of CGC Monte Carlo methods, the MC-KLN [10] and MC-rcBK [3], schematically represented in Fig. (2). Their geometric set up is identical in both cases, differing in the dynamical input for the $\left(x, k_{t}\right)$-dependence of the ugd's, either the KLN model[14] or the rcBK approach. Particle production is then calculated according to $k_{t}$-factorization.

As shown in Fig. (2), MC-CGC approaches systematically yield a larger initial eccentricity than Glauber ones, both at RHIC and LHC energies. However, it is unclear to what extent such is a robust property of the CGC formalism (see for instance the CYM studies in [16]) or an artifact of 

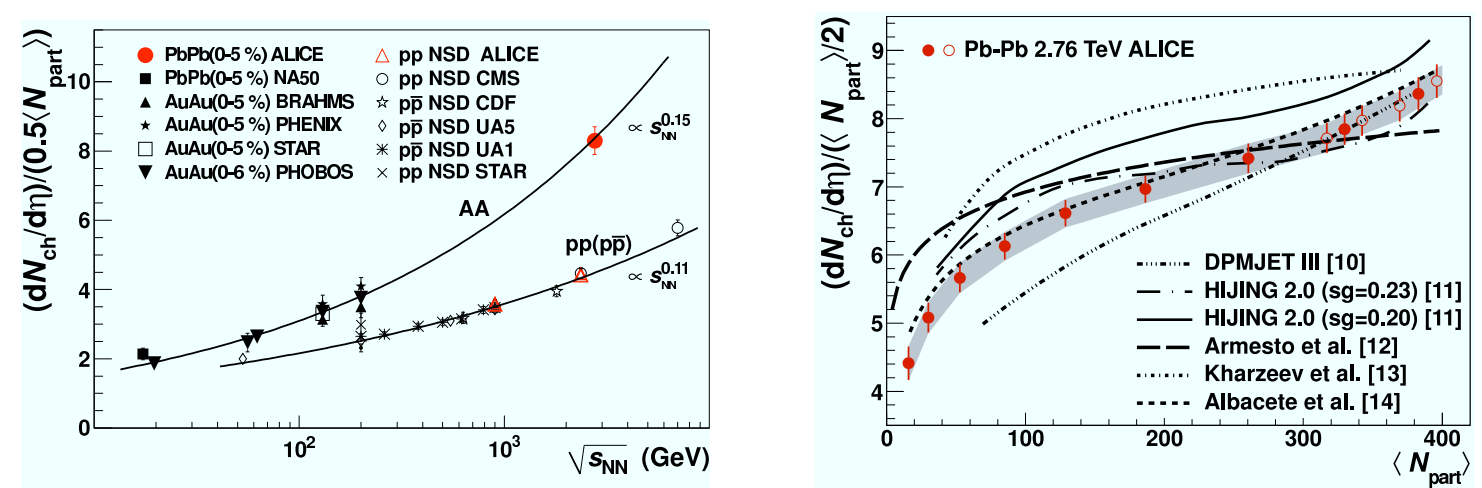

Figure 1: Energy (left) and centrality (right) dependence of mid-rapidity multiplicities in $\mathrm{A}+\mathrm{A}$ and $\mathrm{p}+\mathrm{p}$ collisions. Figures and LHC data by the ALICE collaboration [2, 1].
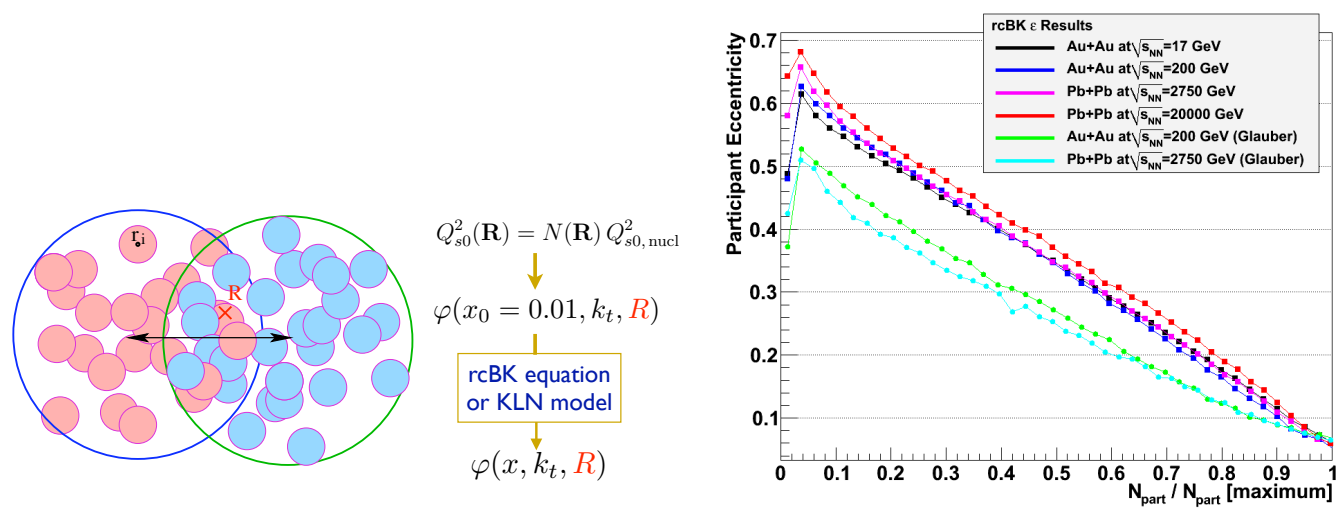

Figure 2: Left: Sketch of the CGC Monte Carlo implementation. Right: Participant eccentricity as a function of centrality for rcBK-CGC and Glauber initial conditions at different collisions energies (courtesy of J. Nagle).

their current implementation in phenomenological works. Also, it should be kept in mind that initial spatial gradients are very sensitive to particle production in the dilute periphery of the collision area. There, the applicability of the CGC formalism, which relies in the presence of high gluon densities, is not guaranteed. Rather, non-perturbative effects may play an important role in that region. In that sense, aspects of the modeling in the MC tools used, such as the relevant sources of fluctuations or the model for the nucleon geometry themselves (e.g thick discs vs. gaussian) may be more relevant for the determination of initial eccentricities than the choice of underlying description for initial particle production, i.e CGC or Glauber.

\section{High $p_{t}$ : Forward particle production at RHIC}

The observation of suppression phenomena in forward measurements in $\mathrm{d}+\mathrm{Au}$ collisions at RHIC has been consistently explained in terms of CGC effects $[6,5]$. Thus, the single inclusive spectra measured both in $\mathrm{p}+\mathrm{p}$ and $\mathrm{d}+\mathrm{Au}$ (Fig. (3)) left) are well described in the framework of the hybrid approach proposed in [11]. Also, the disappearance of forward azimuthal correlations 

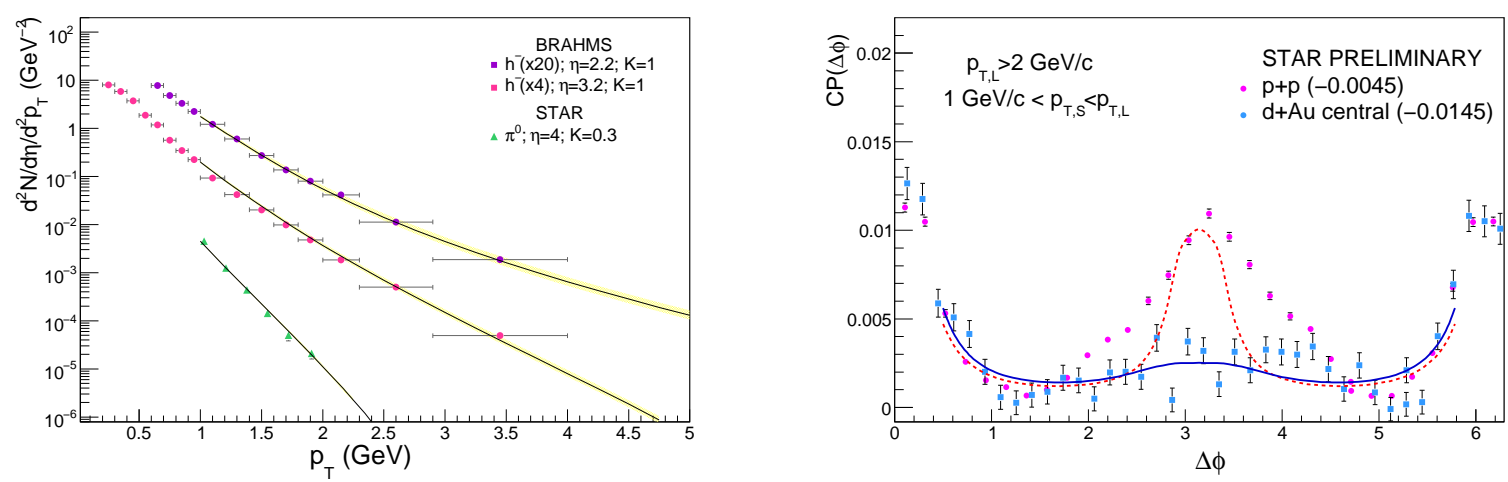

Figure 3: Suppression of forward single inclusive yields (left) and di-hadron azimuthal correlations (right) compared to CGC calculations [6,5]. Data by BRAHMS and STAR collaborations, respectively .

(Fig. (3) right) can be interpreted as due to the presence of a dynamical, semi-hard scale in the nucleus wave function. The use of solutions of the running coupling BK equation to describe the $x$-evolution of the nuclear wave function turns out to be necessary in order to attain a good quantitative description of both observables. A caveat in both calculations is that high- $x$ effects, such as energy loss effects or the effects [15] of multi-parton interactions, argued to be important in the forward region [19], are not taken into account. However, preliminary measurements by the STAR collaboration on neutron tagged events, thus reproducing true $\mathrm{p}+\mathrm{Au}$ collisions, confirm the suppression of azimuthal correlations, thus eliminating the uncertainty on the role of multiparton interactions and lending support to the CGC interpretation. These studies have been taken as a baseline to estimate the nuclear modification factors for single inclusive hadron production at the LHC, predicting a similar suppression in $\mathrm{LHC} \mathrm{p}+\mathrm{Pb}$ collisions at mid rapidity to the one observed in forward rapidities at RHIC. This is in contrast with predictions based on the use of collinear factorization and nuclear pdf's (see eg [18]). However, the predictions of [6] suffer of an uncertainty on the absolute normalization, due to the fact that the nuclear and proton saturation scales were fitted independently to data. The requirement that initial state, saturation effects should disappear at high enough $p_{t}$ demands that they should be related through the thickness function. Such could be achieved by redoing the phenomenological analyses of $[6,5]$ in the MC-CGC set up.

\section{References}

[1] K. Aamodt et al. Centrality dependence of the charged-particle multiplicity density at mid-rapidity in $\mathrm{Pb}-\mathrm{Pb}$ collisions at sqrt $(\mathrm{sNN})=2.76 \mathrm{TeV}$. PRLTA, 106,032301.2011, 106:032301, 2011. Long author list - awaiting processing.

[2] B Abelev et al. Charged-particle multiplicity density at mid-rapidity in central $\mathrm{Pb}-\mathrm{Pb}$ collisions at $\operatorname{sqrt}(\mathrm{sNN})=2.76 \mathrm{TeV}$. Phys. Rev. Lett., 105:252301, 2010 .

[3] Javier L. Albacete and Adrian Dumitru. A model for gluon production in heavy-ion collisions at the LHC with rcBK unintegrated gluon densities. 2010. 
[4] Javier L. Albacete and Yuri V. Kovchegov. Solving high energy evolution equation including running coupling corrections. Phys. Rev., D75:125021, 2007.

[5] Javier L. Albacete and Cyrille Marquet. Azimuthal correlations of forward di-hadrons in d+Au collisions at RHIC in the Color Glass Condensate. Phys.Rev.Lett., 105:162301, 2010.

[6] Javier L. Albacete and Cyrille Marquet. Single Inclusive Hadron Production at RHIC and the LHC from the Color Glass Condensate. Phys. Lett., B687:174-179, 2010.

[7] Nestor Armesto, Carlos A. Salgado, and Urs Achim Wiedemann. Relating high-energy lepton-hadron, proton-nucleus and nucleus-nucleus collisions through geometric scaling. Phys.Rev.Lett., 94:022002, 2005.

[8] Fritz W. Bopp, R. Engel, J. Ranft, and S. Roesler. Inclusive distributions at the LHC as predicted from the DPMJET-III model with chain fusion. 2007.

[9] Wei-tian Deng, Xin-Nian Wang, and Rong Xu. Gluon shadowing and hadron production in heavy-ion collisions at LHC. Phys. Lett., B701:133-136, 2011.

[10] Hans-Joachim Drescher and Yasushi Nara. Eccentricity fluctuations from the Color Glass Condensate at RHIC and LHC. Phys. Rev., C76:041903, 2007.

[11] Adrian Dumitru, Arata Hayashigaki, and Jamal Jalilian-Marian. The color glass condensate and hadron production in the forward region. Nucl. Phys., A765:464-482, 2006.

[12] Francois Gelis, Edmond Iancu, Jamal Jalilian-Marian, and Raju Venugopalan. The Color Glass Condensate. Ann.Rev.Nucl.Part.Sci., 60:463-489, 2010.

[13] L. V. Gribov, E. M. Levin, and M. G. Ryskin. Semihard Processes in QCD. Phys. Rept., 100:1-150, 1983.

[14] Dmitri Kharzeev, Eugene Levin, and Marzia Nardi. Color glass condensate at the LHC: Hadron multiplicities in p p, p A and A A collisions. Nucl. Phys., A747:609-629, 2005.

[15] B. Z. Kopeliovich, J. Nemchik, I. K. Potashnikova, M. B. Johnson, and I. Schmidt. Breakdown of QCD factorization at large Feynman x. Phys. Rev., C72:054606, 2005.

[16] T. Lappi and R. Venugopalan. Universality of the saturation scale and the initial eccentricity in heavy ion collisions. Phys. Rev., C74:054905, 2006.

[17] Larry D. McLerran and Raju Venugopalan. Boost covariant gluon distributions in large nuclei. Phys. Lett., B424:15-24, 1998.

[18] C. A. Salgado et al. Proton-Nucleus Collisions at the LHC: Scientific Opportunities and Requirements. 2011.

[19] Mark Strikman and Werner Vogelsang. Multiple parton interactions and forward double pion production in pp and dA scattering. Phys. Rev., D83:034029, 2011. 